\title{
Knowing power grids and understanding complexity science ${ }^{1}$
}

\author{
M. Rosas-Casals, ${ }^{2 *}$ S. Bologna, ${ }^{3}$ E. Bompard, ${ }^{4}$ G. D'Agostino, ${ }^{3}$ W. Ellens, ${ }^{5,6}$ G. A. \\ Pagani, ${ }^{7}$ A. Scala, ${ }^{8,9}$ T. Verma $^{6}$
}

\begin{abstract}
Complex networks theory has been well established as a useful framework for studying and analyzing structure, dynamics and evolution of many complex systems.

Infrastructural and man-made systems like power grids, gas and water networks and the internet, have been also included in this network framework, albeit sometimes ignoring the huge historical body of knowledge surrounding them. Although there seems to exist clear evidence that both complexity approach in general, and complex networks in particular, can be useful, it is necessary and profitable to put forward some of the limits that this scheme is facing when dealing with not so complex but rather complicated systems like the power grid. In this position paper we offer a critical revision of the usefulness of the complexity and complex networks' approach in this later case, highlighting both its strengths and weaknesses. At the same time we emphasize the disconnection between the so called complex and the more traditional engineering communities as one of the major drawbacks in the advent of a true body of understanding (more than simply knowing) the subtleties of this kind of complex systems.
\end{abstract}

\section{Engineered systems and complexity}

Complexity can be considered a relatively new and basically interdisciplinary field that deals with the so called complex systems (Érdi 2007, Gros 2008). It is worth to stress that although experimental observations are possible, these are usually not considered a part of the field of complex systems itself, which is primarily devoted to theoretical developments (Newman 2011). Although there is no general agreement on a strict

\footnotetext{
${ }^{1}$ This research grew out and includes the final summary of the SESAME (Securing the European Electricity Supply Against Malicious and accidental thrEats) workshop held at the $7^{\text {th }}$ International Conference on Critical Information Infrastructures Security (CRITIS 2012), on September $18^{\text {th }}$, in Lillehammer (Norway).

2* Sustainability Measurement and Modelling Lab., Universitat Politècnica de Catalunya-Barcelona Tech, Terrassa (Spain). To whom correspondence should be addressed. Email: rosas@mmt.upc.edu

${ }^{3}$ National Agency for New Technologies, Energy and Sustainable Economic Development-ENEA, Bologna (Italy)

${ }^{4}$ Joint Research Centre, Institute for Energy and Transport, Petten (The Netherlands)

${ }^{5}$ Dutch Organization for Applied Scientific Research-TNO, The Hague (The Netherlands)

${ }^{6}$ Delft University of Technology -TU Delft, Delft (The Netehrlands)

${ }^{7}$ Johann Bernoulli Institute for Mathematics and Computer Science, University of Groningen, Groningen (The Netherlands)

${ }^{8}$ ISC-CNR Physics Dept., Univ. "La Sapienza", Roma (Italy)

${ }^{9}$ London Institute of Mathematical Sciences, Mayfair, London (United Kingdom)
} 
definition of a "complex system", these are usually composed of many interacting units often called "agents" (which can be consumers, species, vehicles, neurons, etc.) capable of many different types of interactions (Nature 2008). This definition is usually completed with that of emergent behavior, so that the collective behavior of those parts together is more than the sum of their individual ones. These emergent phenomena are usually related with nonlinear positive and negative feedback loops among composing parts (which means that small changes in the initial conditions can have a big effect at a global level) and the appearance of self-organizing processes, which tends to drive them out of any perennial static equilibrium (i.e., classical thermodynamic principles do not apply). Classic and so-believed examples of complex systems include ecosystems, insect colonies, human societies, financial markets and infrastructural systems like the Internet, road networks, gas pipe work or the power grid.

In the particular later context of power networks, complexity appears as hotchpotch where many different concepts from complexity science have been applied and analyzed. Efforts involved in trying to characterize the power grid as a complex system have revolved essentially around the following issues [see (Mei, Zhang et al. 2011) for a complete review and references therein]:

\section{Criticality}

As said before, self-organizing processes are one usual outcome of complex systems. In any self-organizing process, a pattern appears that can be clearly recognized and, far from subjective opinions, statistically quantified. Among the many statistical signatures of self-organization, those involved in the characterization of phase-transitions and critical states have become the most remarkable, like scaling relations, power-law divergences of some quantities, universality, fractality, etc. Notably, the appearance of algebraic probability distributions, especially power laws, in terms of event or object sizes, is often thought to be the signature of hierarchy, robustness, criticality and universal underlying mechanisms (Bak 1996). In the case of power transmission networks, time series of usual measures of blackout size like energy not served, power loss or number of customers affected, have been shown to be algebraically distributed in many countries. This apparent ubiquitous evidence have led to believe and try to demonstrate that power systems tend to self-organize near a critical point and that there may be some universality ruling the inner depths of these systems [see (Dobson, Carreras et al. 2007) for a complete review and references therein].

\section{Complex networks}

In the last years, complex networks theory has appeared as a new framework to study complex systems (Newman 2010). A network is a simplified representation of a system. It reduces it to a graph, an abstract entity that captures essentially its structure. Although some amount of specificity is lost, this is an advantage when questions that arise from difficult hypothesis can be answered with limited computational power and simplifying assumptions. Complex networks' theory applied to infrastructures in general, and power 
grid systems in particular, has produced a huge amount of literature basically centered around the three following issues:

- Structure. In order to understand the structure of the interactions between the components of a network, much effort has been done in defining metrics to characterize the topology of real networks [see (Pagani, Aiello 2011) for a complete review and references therein]. Some of them, such as the degree (i.e., number of edges connecting an element to others), degree probability distribution, clustering coefficient (i.e., number of connected triples) and average path length (i.e., number of edges needed to travel from one element of the graph to another) have been used thoroughly in order to identify unifying principles and common statistical properties which, in turn, has allowed the development of models of networks (Boccaletti, Latora et al. 2006). A complete characterization of a network's topology is motivated by the expectancy that modeling the structure of a complex network would lead to a better understanding of its evolutionary mechanisms and to a better comprehension of its dynamical and functional behavior (Solé, Ferrer i Cancho et al. 2003). In the case of power grids, some of the questions addressed with the analysis of the structure of their topology have tried to answer which are the measures that best characterize it and whether they are useful in order to understand and classify these systems. If topology and dynamics are deeply interwoven, another important question is whether we can find evidences of "weak" (or conversely "robust") topologies and how different topologies stand the impact of different kinds of damages. This last aspect is essential for a critical infrastructure as the power grid.

- Dynamics. In the case of complex networks, dynamics refers to the case in which the processes involved in the redistribution of flows are taken into account. It involves the modeling of flows of the physical quantities of interest over the network. In power networks it has been basically used to model blackouts and the robustness of the network to withstand malfunctions and attacks (Albert, Jeong et al. 2000, Motter, Lai 2002). When it comes to modeling the dynamics, the situation is far more complicated since the components of a network may have different dynamical behaviors and flows are often a highly variable quantity, both in space and time. The usual shortcut to overcome these difficulties has been the assumption of a characteristic load of an element as a measure of its capacity and the association of this load to a topological measure defined on it. The most used topological measure has been one standard measure of node centrality (Boccaletti, Latora et al. 2006): the so called betweenness centrality of that element (i.e., how many shortest paths cross that particular node or edge). The robustness of the network has been analyzed by means of cascading processes, where an element of the system, characterized by a finite capacity (i.e., the maximum load that this element can handle and directly correlated to its topological value of betweenness) is eliminated. This fact 
changes the shortest paths between nodes and, consequently, the distribution of loads, creating overloads on some other nodes that, similarly, are eliminated from the network. This leads to a new redistribution of loads and the new overloaded nodes are removed. The redistribution process continues until when, at a certain time, all the remaining nodes have values under or equal to its capacity. Somehow related to this analysis, there has been lately remarkable research in network synchronization, where networks of oscillators with particular distributions of frequencies are interpreted as models of frequency synchronization dynamics among generators and loads. (Lozano, Buzna et al. 2012)

- Evolution. In analyzing topology and dynamics of any networked system, we usually can only see the present outcome of a huge evolving process taking place in multiple spatial and temporal scales. Such process is driven by multiple and, usually, unknown forces. As these unknown forces shape and, at the same time, are being shaped by the evolving structure and dynamics of the network, its growth can be an intricate process. Here dynamics impels new topological forms that, at the same time, modify the flows of information between its constituents. Therefore, in order to fully comprehend a network we must deal with its evolution, as well as with its structure and topology. Unlike social networks for example, where topologies and dynamics can be easily retrieved from past accumulated data (Kumar, Novak et al. 2006, Leskovec, Kleinberg et al. 2007), information regarding past evolutionary stages in infrastructural systems is difficult to obtain and usually not available at all. This drawback complicates the processes of corroborating assumptions imposed by models or validating the appearance of other intermediate evolutionary processes. With very few exceptions, like in (Buzna, Issacharoff et al. 2009), very little is known about the evolution of infrastructural networks. Its study is an essential component of the complex networks research agenda in order to shed light on some fundamental questions. As networked systems grow by adding elements and, at the same time, by coupling their dynamics to those already present, the level of interaction between elements varies and this process modifies the fluxes of information and energy flows. Infrastructure networks in general (and power grids in particular) belong to the engineering field, where objectives and constraints are clear a priori. However, most technological networks have been continuously going through changes, spanning and crossing urban and natural systems from their early stages, adapting and being adapted by human societies, landscapes, territories and other constraints. This adaptive process has the power to modify the initial objective functions.

\section{Complex or complicated?}

There are huge conceptual and practical problems when engineering and complexity sciences are tried to be connected. The truth is that finding most of the characteristics of 
complex systems in man-made ones like the power grid is a difficult task. Concepts like emergence, self-organization and adaptation are far from the engineering purposes and difficult, if not impossible, to experience in the man-made realm. Engineering is about assembling pieces that work in specific ways: that is, designing (complicated or not so) systems. These systems are robust in terms of redundancies but not adaptation: once a transmission line is constructed, it remains there for years to come and the system must deal with its fixed topology, and associated impact in the system's dynamics, whether it is beneficial for the whole functioning scheme or not in the long term. In the power grid case, self-organization does not exist: synchronization and phase angles must be maintained constantly under schedule. And so, it cannot lead to any kind of emergent behavior or pose the system to any critical state (if we consider blackouts not as an emergent phenomenon but as an expected malfunction output and so, understandable from the individual behaviors of the system's composing elements).

Complexity and network theory have offered a new and fresh approach to an old system, that of the power grid. However, and paradoxically, it seems its usefulness has been relatively undermined by its oversimplifying assumptions. From a general conceptual point of view, there is one main drawback associated to the oversimplification of any real power network. This is that for power grids we can (a) obtain a closed form mathematical model of the many equations that assess the physical system and (b) manage to solve it in the time it is needed (being it off-line or even online). Thus, the question about why do we need simplified (or approximated) complex networks models if we can get better answers with fully precise models seems a natural and obvious one. The approach offered by complex networks theory, with purely topological analysis (or even extended ones to take into account minimal electrical information), has been useful in order to detect critical components and evaluate topological robustness. The assessment of these results has been done by means of correlating these topological and extended degrees (Bompard, Napoli et al. 2009) with load shedding in real systems (over several time spans) or even with statistical failure data of real blackouts and failures (Solé, Rosas-Casals et al. 2008). However results have been not conclusive and their applicability could not be generalized. Graph theory and applied statistical physics alone cannot account for a full characterization of an electricity grid: there is a need for the deeper interplay between structural and electrical aspects.

As far as the dynamics is concerned, it is important here to stress the meaning of "dynamical behavior" (or simply dynamics) when dealing with complex networks, in contrast with its meaning in the power generation, operation and control field. While complex networks' dynamics is related to the flow of information, energy or matter through the networked system and the different temporal values that characterize the resulting feature vector, power systems' dynamics is related to frequency, synchronization, swings and transient stability performance. If the word dynamics is used as in the complex networks acceptation, power grids seem to be an optimal candidate for the kind of dynamic analysis presented above, since cascading failures 
have been usually the prologue to huge blackouts (Nedic, Dobson et al. 2006). But at the same time, power system operation, flow and generation turns out to be one of the most mathematically complicated problems encountered in engineering nowadays. All the variables and processes involved in such calculations demonstrate that power grids do not concentrate flows depending on topological centrality measures (like betweenness) nor the pool of sinks and sources are necessarily constrained by shortest paths (Wood, Wollenberg 1996). Therefore, most of the initial complex networks dynamic models encountered in the literature have only a qualitative role as explanatory theory. Although they provide some indications on the actions that can be performed in order to decrease undesired effects such as congestions, avalanches of node breakdown and cascading failures, these are not realistic neither sufficiently accurate to explain the power grid dynamics. If the word dynamics is used as in the engineering acceptation, it does not mean a change in the configuration or topology (and not even the power flows over the lines). Dynamics is referred to the voltage and angular behavior over time which concerns on stability. It does not mean a change in the configuration, but on the voltage and angular behavior over time. Therefore, blackouts are due to voltage and angular instability and cannot be explained by any of the previous approaches.

It seems reasonable to ask ourselves whether the power grid is only complicated or there are other aspects that can turn it into a complex system. The answer is that maybe the physical network is not complex, but when other layers of agents are present, an increased number of nonlinear interactions and unexpected phenomena can easily arise. These new agents, different from solely transformers, substations or lines, can be multiple heterogeneous decision makers at national and transnational levels, like TSO's (each one operating a subsystem of a global interconnected transmission system), policy makers, regulators or market participants. Topology (and the complex networks approach) appears then as just one more of the many dimensions of performance that influence global risk and security. In addition to purely electrical dynamics, the specific operative status of the grid is then a multivariable and multileveled problem, with possibilities for emergent phenomena and self-organizing processes to develop.

\section{From knowledge to understanding}

There exists an important distinction between knowledge and understanding. While the former is usually constituted by sets of data, facts, observations or behaviors, the later appears when we appreciate that two or more very different-seeming phenomena can be treated as similar in some way (Lander 2010). Complexity science has allowed the creation of understanding out of validated bits of knowledge and there seems to be no doubt about the usefulness of some of its concepts for understanding and optimizing engineered systems (Ottino 2004). In the particular case of power grids, the challenges are basically two: (a) to make useful models and theories that come from simplified schemes and (b) to detect how far we can go in elucidating new metrics for addressing dynamical properties of the system's components, being them transformers, lines or users. Some of the engineering areas where complexity concepts applied to power 
systems are useful and with a high potential of developing sound scientific frameworks in the short and mid terms would be the following:

- Smart Grids. Complex networks approach has been extremely useful in modeling and classifying topologies of real networks (Albert, Barabási 2002). Some topologies are shown to be more efficient than others when information, resources or energy transportation are taken into account as fluxes (Pagani, Aiello 2011). On the other hand, some biologically inspired mathematical models have captured the basic dynamics of network adaptability and have produced solutions with properties comparable to or better than those of realworld infrastructure networks (Tero, Takagi et al. 2010). Such models may provide a useful starting point to improve routing protocols and topology control for infrastructural networks. This can be applied, for example, as a tool to support the modeling of low and medium voltage smart grids or distribution networks designed from scratch. (Pagani, Aiello 2012)

- Agents. It has been previously stated that, in the particular field of power systems, complexity appears when users of many kind interact with the physical system. When interacting and thoughtful agents appear, dependencies among the elements become increasingly important, stochastic processes arise and we are finally dealing with adaptive systems, usually modeled by computational units termed agents (Miller, Page 2007, Railsback, Grimm 2012). Agent (or individual) based modeling has been traditionally linked to complexity and computer sciences and allows to modeling unique and autonomous entities that usually interact with each other and their environment locally. Using agentbased modeling lets us address problems that concern adaptive behavior and emergence phenomena, very much connected to prosumers' (i.e., small producers and consumers of energy at the same time) activities and dynamic behavior of the power grid.

- Interdependent networks. Power systems are the underlying support of most infrastructural systems. Malfunctions and failures in the former dramatically affect the rest of interconnected networks. When interdependent networks are taken in consideration, complexity and complex networks approaches could be once again useful. There is a need for new robustness measures that capture not only purely topological aspects, but also the functionality of the system as a whole. And these approaches are useful in capturing fundamental differences between real loads and commonly used load estimators, meta-vulnerabilities (Kurant, Thiran 2006), or developing analytical models to study cascading failures in interdependent networks (Newman, Nkei et al. 2005, Buldyrev, Parshani et al. 2010, Brummitt, D’Souza et al. 2012).

Above all these issues, there is a pressing requirement of communication among scholars and academic traditions. Results from complex approximations have to be checked with real power networks' operators and facilities. Interaction and 
collaboration between complexity and complex networks communities, on the one hand, and electrical engineers, on the other, is compulsory. In order to fully develop the former issues, where complexity science can clearly help engineering science and conversely, this communicative gap between these two communities of knowledge must be overcome. This is the only way to finally become communities of understanding.

\section{References}

ALBERT, R. and BARABÁSI, A., 2002. Statistical Mechanics of Complex Networks. Reviews of Modern Physics, 74(1), pp. 47-97.

ALBERT, R., JEONG, H. and BARABÁSI, A., 2000. Error and attack tolerance of complex networks. Nature, 406, pp. 378-382.

BAK, P., 1996. How nature works : the science of self-organized criticality. New York, NY, USA: Copernicus.

BOCCALETTI, S., LATORA, V., MORENO, Y., CHAVEZ, M. and HWANG, D.-., 2006. Complex networks: Structure and dynamics. Physics Reports, 424(4-5), pp. 175308.

BOMPARD, E., NAPOLI, R. and XUE, F., 2009. Analysis of structural vulnerability in power transmission grids. International Journal of Critical Infrastructure Protection, 2(1-2), pp. 5-12.

BRUMMITT, C.D., D’SOUZA, R.M. and LEICHT, E.A., 2012. Suppressing cascades of load in interdependent networks. Proceedings of the National Academy of Sciences, 109(12), pp. E680-E689.

BULDYREV, S.V., PARSHANI, R., PAUL, G., STANLEY, H.E. and HAVLIN, S., 2010. Catastrophic cascade of failures in interdependent networks. Nature, 464, pp. 1025-1028.

BUZNA, L., ISSACHAROFF, L. and HELBING, D., 2009. The evolution of the topology of high-voltage electricity networks. International Journal of Critical Infrastructures, 5(1/2), pp. 72-85.

DOBSON, I., CARRERAS, B., LYNCH, V. and NEWMAN, D., 2007. Complex systems analysis of series of blackouts: Cascading failure, critical points, and selforganization. Chaos: An Interdisciplinary Journal of Nonlinear Science, 17(2), pp. 026103.

ÉRDI, P., 2007. Complexity explained. Berlin: Springer.

GROS, C., 2008. Complex and adaptive dynamical systems : a primer. Berlin: Springer. 
KUMAR, R., NOVAK, J. and TOMKINS, A., 2006. Structure and evolution of online social networks, Proceedings of the 12th ACM SIGKDD international conference on Knowledge discovery and data mining 2006, ACM, pp. 611-617.

KURANT, M. and THIRAN, P., 2006. Layered complex networks. Physical Review Letters, 96(13), pp. 138701-138701.

LANDER, A., 2010. The edges of understanding. BioMed Central Biology, 8(1), pp. 40.

LESKOVEC, J., KLEINBERG, J. and FALOUTSOS, C., 2007. Graph Evolution: Densification and Shrinking Diameters. ACM Transactions on Knowledge Discovery from Data, 1(1),.

LOZANO, S., BUZNA, L. and DIAZ-GUILERA, A., 2012. Role of network topology in the synchronization of power systems. The European Physical Journal B Condensed Matter and Complex Systems, 85(7), pp. 1-8.

MEI, S., ZHANG, X., CAO, M. and SPRINGERLINK (ONLINE SERVICE), 2011last update, Power grid complexity [Homepage of Tsinghua University Press ; Springer], [Online].

MILLER, J.H. and PAGE, S.E., 2007. Complex Adaptive Systems. An Introduction to Computational Models of Social Life. Princeton: Princeton University Press.

MOTTER, A.E. and LAI, Y., 2002. Cascade-based attacks on complex networks. Phys. Rev. E, 66(065102(R)),.

NATURE, 2008. Language: Disputed definitions. 455, pp. 1023-1028.

NEDIC, D.P., DOBSON, I., KIRSCHEN, D.S., CARRERAS, B.A. and LYNCH, V.E., 2006. Criticality in a cascading failure blackout model. International Journal of Electrical Power \& Energy Systems, 28(9), pp. 627-633.

NEWMAN, D.E., NKEI, B., CARRERAS, B.A., DOBSON, I., LYNCH, V.E. and GRADNEY, P., 2005. Risk assessment in complex interacting infrastructure systems, HAWAII INTERNATIONAL CONFERENCE ON SYSTEM SCIENCES 2005, IEEE.

NEWMAN, M.E.J., 2010. Networks : an introduction. Oxford; New York: Oxford University Press.

NEWMAN, M., 2011. Resource Letter CS-1: Complex Systems. American Journal of Physics, 79(8), pp. 800-810.

OTTINO, J.M., 2004. Engineering complex systems. Nature, 427(6973), pp. 399-399.

PAGANI, G.A. and AIELLO, M., 2012. Power Grid Network Evolutions for Local Energy Trading. ArXiv preprint arXiv:1201.0962, .

PAGANI, G.A. and AIELLO, M., 2011. The Power Grid as a Complex Network: a Survey. ArXiv e-prints, . 
PAGANI, G.A. and AIELLO, M., 2011. Towards Decentralization: A Topological Investigation of the Medium and Low Voltage Grids. Smart Grid, IEEE Transactions on, 2(3), pp. 538-547.

RAILSBACK, S.F. and GRIMM, V., 2012. Agent-Based and Individual-Based Modeling. Princeton: Princeton University Press.

SOLÉ, R., FERRER I CANCHO, R., MONTOYA, J.M. and VALVERDE, S., 2003. Selection, tinkering and emergence in complex networks. Complexity, 8(1), pp. 20-33.

SOLÉ, R., ROSAS-CASALS, M., COROMINAS-MURTRA, B. and VALVERDE, S., 2008. Robustness of the European power grids under intentional attacks. Physical Review E, 77(2), pp. 026102.

TERO, A., TAKAGI, S., SAIGUSA, T., ITO, K., BEBBER, D.P., FRICKER, M.D., YUMIKI, K., KOBAYASHI, R. and NAKAGAKI, T., 2010. Rules for Biologically Inspired Adaptive Network Design. Science, 327(5964), pp. 439-442.

WOOD, A.J. and WOLLENBERG, B.F., 1996. Power generation, operation and control. New York: John Wiley \& Sons. 